\title{
Primary Central Nerve System Lymphoma Affecting the Lumbosacral Nerve Root: A Case Report
}

\author{
Junhyung Kim, Bonggyu Ryu, Seung-Jae Hyun, Ki-Jeong Kim \\ Department of Neurosurgery, Spine Center, Seoul National University Bundang Hospital, Seoul National University \\ College of Medicine, Seongnam, Republic of Korea \\ Corresponding author: K-Jeong Kim \\ Department of Neurosurgery, Spine Center, \\ Seoul National University Bundang Hospital \\ Seoul National University College of Medicine, \\ 82, Gumi-ro 173beon-gil, Bundang-gu, \\ Seongnam 13620, Republic of Korea \\ Tel: +82-31-787-7169 \\ Fax: $+82-31-787-4097$ \\ E-mail: kijeong@snu.ac.kr \\ The cauda equina is a rare location for malignant tumors. This report presents a case \\ of cauda equina lymphoma and a review of the related literature. A 67-year-old female \\ presented with a one-month history of intractable pain in the right lower extremity. \\ Imaging studies revealed an intradural mass at the L4-5 vertebral body level. Surgical \\ treatment was performed for neural decompression and biopsy. The ventral branch \\ of the right S1 nerve root was particularly affected by the tumor. Histopathologic exam \\ confirmed diffuse large B-cell lymphoma, and the patient has been diagnosed as primary \\ central nerve system lymphoma.
}

Received: August 10, 2020

Revised: September 4, 2020

Accepted: September 5, 2020
Key Words: B-cell lymphoma; Cauda equina; Lumbosacral region; Neurolymphomatosis

\section{INTRODUCTION}

The cauda equina is a rare location for neoplasms, accounting for $0.5 \%$ to $5.3 \%$ of all benign and malignant tumors of the spinal cord and appendage, ${ }^{6,8)}$. The most common pathology of the cauda equina tumor includes myxopapillary ependymoma, often involving the filum terminale or the conus medullaris, and schwannoma, which could occur in any peripheral sensory nerve. Malignant tumors are extremely uncommon in the cauda equina, and, to the best of our knowledge, there have been only a few reported cases of metastasis or lymphoma.

In this article, we present an unusual case of primary central nervous system (CNS) lymphoma that occurred in the cauda equina as the first manifestation. This lymphoma involved both the central and peripheral nerve systems without evidence of systemic disease, which is a particularly rare occurrence.

\section{CASE REPORT}

A 67-year-old female presented with intractable pain in the right lower leg a month prior to her visit. Her symptom was accompanied by gait disturbance due to weakness in both legs, but dominant on the right side. She also experienced urinary frequency and incontinence, likely indicating cauda equina syndrome. On neurologic examination, right ankle plantar flexion was slightly impaired as Medical Research Council grade $4 / 5$, and paresthesia was dominantly distributed in the right
L5-S1 dermatome. On magnetic resonance imaging (MRI), multiple intradural nodular lesions at the L3 to S3 vertebral body level were found, possibly suggesting a benign peripheral nerve sheath tumor, such as schwannomatosis (Fig. 1). Additional findings included abnormal contrast enhancement in the conus medullaris, which was interpreted as spinal cord infarction

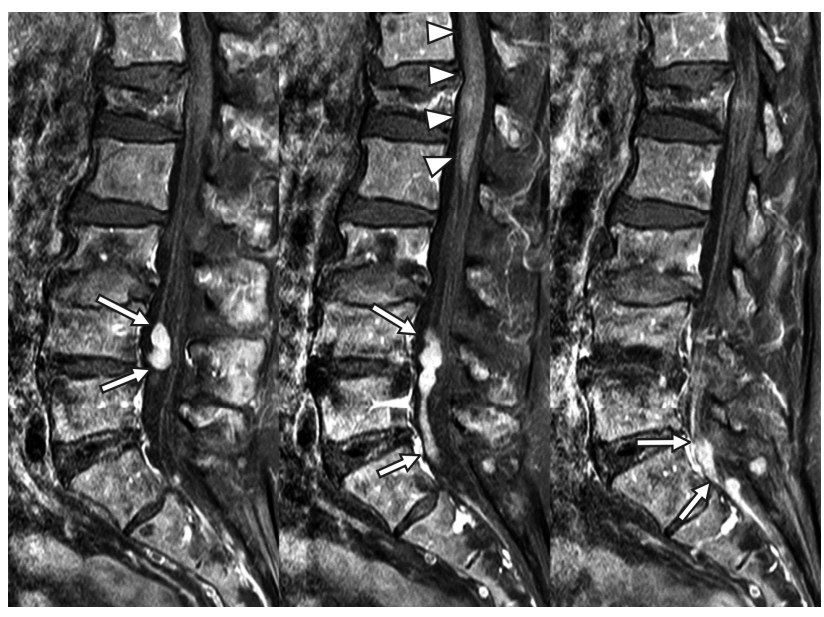

Fig. 1. Lumbosacral magnetic resonance imaging showing the intradural tumor in the cauda equina. Multiple enhancing nodules in the cauda equina along the L3 to S3 vertebral body level are observed in the sagittal sections of post-contrast $\mathrm{Tl}$-weighted images. The largest lesion with a rosary pattern at the $L 4-5$ vertebral body level is $1.1 \times 0.9 \mathrm{~cm}$ in size, extending to the right $\mathrm{S} 1$ neural foramen (arrows). Atypical contrast enhancement in the conus medullaris is also observed (arrowheads). 
regarding the patient's prior history of spinal trauma with vertebral compression fracture at L1. Routine laboratory examination, including serology, showed no specific findings; however, interestingly, serum lactic acid dehydrogenase level was slightly increased as $256 \mathrm{IU} / \mathrm{L}$ while other inflammatory markers were normal.

The patient underwent surgical treatment for decompression of the space-occupying lesion and tumor biopsy. We planned to remove the largest mass at the vertebral body level, which was most likely to be the cause of the patient's symptoms. After L4 and L5 laminotomy, the tumor was sufficiently exposed within the subarachnoid space. The largest mass with a rosary pattern particularly involved the ventral branch of the right S1 nerve root, compressing adjacent nerve roots in the cauda equina (Fig. 2A). Similar tumorous changes were observed in the ventral branch of the right S2 nerve root, whereas the branches
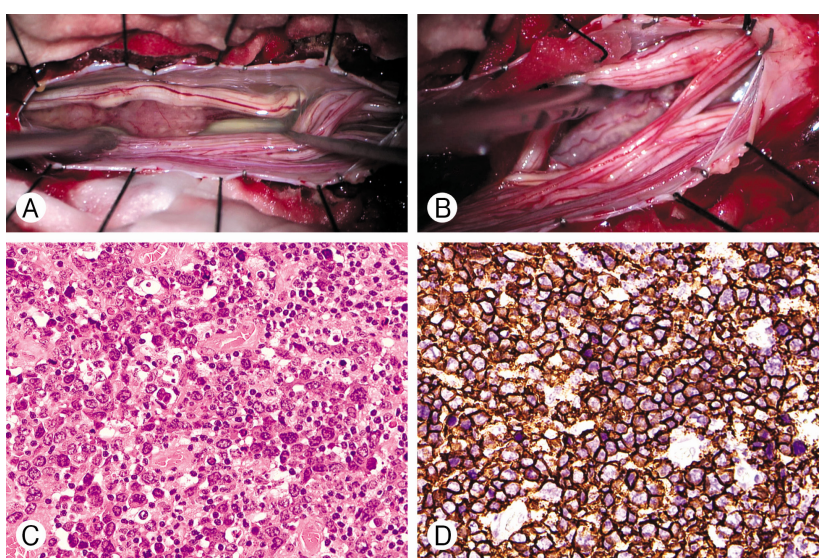

Fig. 2. Intraoperative and histopathologic findings. A dumbbell-shaped mass is involving one of the lumbosacral nerve roots $(A)$, which is identified as the ventral branch of the right $\mathrm{S} 1$ nerve root passing through its designated foramen (B). The main mass is exposed after microscopic dissection and totally resected. High-power magnification view (hematoxylin and eosin stain [H\&E], $\times 400$ magnification) section. (C) reveals diffuse infiltration by large, atypical lymphoid cells, which are positive on CD20 immunostaining (D).
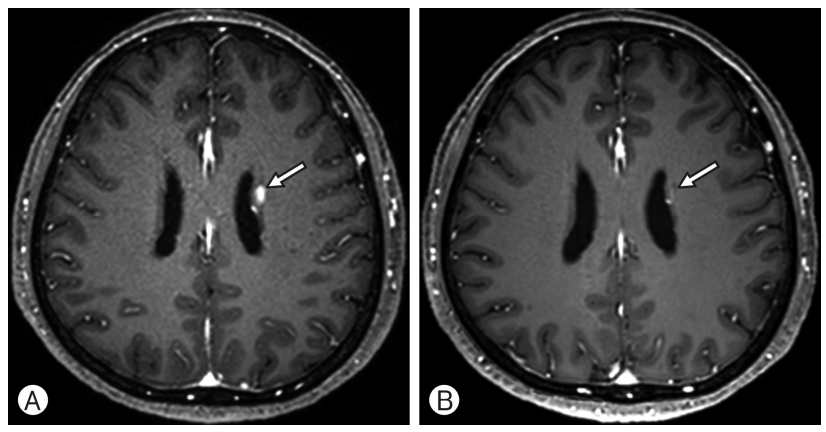

Fig. 3. Brain magnetic resonance imaging showing an enhancing nodule in the left paraventricular white matter. This lesion is measured $8 \mathrm{~mm}$ in the post-contrast $\mathrm{Tl}$ axial image (A) and disappeared in the follow-up after 2 cycles of chemotherapy (B). of the right L5 nerve root appeared to be normal (Fig. 2B). A frozen section was obtained from the core of the main mass and reported as a poorly differentiated malignant tumor rather than schwannoma. Since the tumor was ill-defined so that the nerve fibers within the nerve root were not clearly demarcated during microscopic dissection, we resected the entire lesion. The patient was recovered without any complications or neurologic deterioration, and her intractable pain, as well as gait disturbance and urinary symptoms, was significantly improved after surgery.

The pathology report from the permanent specimen confirmed diffuse large B-cell lymphoma (BCL) that CD20 and BCL6 were positive in immunohistochemistry (Fig. 2C, D). Wholebody and brain $18 \mathrm{~F}$-fluorodeoxyglucose positron emission tomography (PET)/computed tomography (CT) was performed, which was negative for malignancy in other sites except the lumbosacral spine. Otherwise, brain MRI revealed a small lesion in the left frontal paraventricular white matter near the body of the caudate nucleus (Fig. 3A), providing evidence of CNS involvement, together with the lesion in the conus medullaris as shown above (Fig. 1). These findings indicate that this lymphoma affected both the central (paraventricular and intramedullary) and peripheral (cauda equina) nerve systems at once. After the diagnosis of primary CNS lymphoma, the patient had subsequently started systemic chemotherapy with rituximab, high-dose methotrexate, procarbazine, vincristine regimen. The patient was well-tolerated on the treatment, and the extent of lesions in the conus medullaris and the caudate nucleus were both decreased on follow-up MRI for early assessment after 2 cycles of chemotherapy (Fig. 3B).

\section{DISCUSSION}

Neurolymphomatosis is a rare entity of lymphoma that affects the peripheral nerve system ${ }^{1)}$. Despite its rarity, there have been similar reports of neurolymphomatosis arising from the lumbosacral nerve roots in the cauda equina (Table 1). These tumors have been categorized as primary cauda equina lymphoma by some authors ${ }^{10,14,15)}$. Additionally, a few reports demonstrated secondary involvement in the cauda equina accompanied by lymphoma in other sites, which is usually refractory and associated with poor outcome ${ }^{2,3,13)}$.

Based on the literature review, primary cauda equina lymphoma has been frequently misdiagnosed as other spinal tumors or inflammatory diseases ${ }^{4,11,13)}$. PET/CT appears to be an important diagnostic procedure, as neurolymphomatosis often affects multiple sites ${ }^{7}$. Cerebrospinal fluid exam is inconsistent in previous reports, thus less useful ${ }^{5,14)}$. Surgical intervention is a definite method for the diagnosis and is highly recommended if other modalities are inconclusive.

Regarding the treatment, appropriate evaluation for systemic or CNS involvement is essential. Additional thin-section contrast-enhanced brain MRI is helpful because PET/CT is often 
negative as in our case. As neurolymphomatosis may involve the arachnoid space, the regimen based on protocols targeting
CNS involvement has often been chosen". Although the general outcome of neurolymphomatosis in the cauda equina remains

Table 1. Previously reported cases presenting with isolated neurolymphomatosis in the cauda equina in the last decade

\begin{tabular}{|c|c|c|c|c|c|c|c|c|c|c|c|}
\hline References & Year & $\begin{array}{l}\text { Age/ } \\
\text { Sex }\end{array}$ & $\begin{array}{c}\text { Major } \\
\text { symptom }\end{array}$ & $\begin{array}{l}\text { Vertebral } \\
\text { level }\end{array}$ & Biopsy & $\begin{array}{l}\text { Pathologic } \\
\text { subtype }\end{array}$ & $\begin{array}{l}\text { Primary or } \\
\text { secondary }\end{array}$ & $\begin{array}{l}\text { Systemic } \\
\text { evaluation }\end{array}$ & $\begin{array}{c}\text { CNS } \\
\text { evaluation }\end{array}$ & Treatment & Outcome \\
\hline $\begin{array}{l}\text { Imasaki } \\
\text { et al. }\end{array}$ & 2012 & $69 / \mathrm{M}$ & $\begin{array}{l}\text { Lumbosacral } \\
\text { radiculopathy }\end{array}$ & T12-L1 & $\begin{array}{l}\text { Open biopsy } \\
\text { at T12-L1 }\end{array}$ & DLBCL & Primary & $\begin{array}{l}\text { Negative on } \\
\text { whole-body } \\
\mathrm{CT} \text { and } \\
\text { bone scan }\end{array}$ & - & HD-MTX & $\begin{array}{l}\text { Progression-free } \\
\text { for } 1 \text { year, } \\
\text { deceased in } 18 \\
\text { months }\end{array}$ \\
\hline $\begin{array}{l}\text { Nishida } \\
\text { et al. }\end{array}$ & 2012 & $\begin{array}{l}47 / \\
M\end{array}$ & $\begin{array}{l}\text { Lumbosacral } \\
\text { radiculopathy }\end{array}$ & - & $\begin{array}{l}\text { CSF exam } \\
\text { only }\end{array}$ & $\mathrm{DLBCL}$ & Primary & $\begin{array}{l}\text { Negative on } \\
\text { whole-body } \\
\text { CT }\end{array}$ & $\begin{array}{l}\text { Positive } \\
\text { on CSF } \\
\text { cytology }\end{array}$ & $\begin{array}{l}\text { HD-MTX, } \\
\text { Ara-C, } \\
\text { RT } 40 \text { Gy }\end{array}$ & $\begin{array}{l}\text { Alive, } \\
\text { progression-free } \\
\text { for } 1.5 \text { years }\end{array}$ \\
\hline Teo et al. & 2012 & $58 / \mathrm{M}$ & $\begin{array}{l}\text { Lumbosacral } \\
\text { radiculopathy }\end{array}$ & T11-L4 & Open biopsy & DLBCL & Primary & - & $\begin{array}{l}\text { Negative } \\
\text { on CSF } \\
\text { cytology }\end{array}$ & N/A & $\begin{array}{l}\text { Alive, } \\
\text { progression-free } \\
\text { for } 2 \text { years }\end{array}$ \\
\hline Tsai et al. & 2013 & $62 / F$ & $\begin{array}{l}\text { Lumbar } \\
\text { radiculopathy }\end{array}$ & L3-4 & $\begin{array}{l}\text { Open biopsy } \\
\text { at L3-4 }\end{array}$ & DLBCL & Primary & - & - & - & - \\
\hline \multirow[t]{2}{*}{$\begin{array}{l}\text { Broen } \\
\text { et al. }\end{array}$} & 2014 & $75 / F$ & $\begin{array}{l}\text { Lumbosacral } \\
\text { radiculopathy }\end{array}$ & $\llcorner 4-5$ & $\begin{array}{l}\text { Biopsy of } \\
\text { right } L 5 \\
\text { nerve root }\end{array}$ & DLBCL & Primary & - & $\begin{array}{l}\text { Positive } \\
\text { on CSF } \\
\text { cytology }\end{array}$ & - & $\begin{array}{l}\text { Deceased in } 10 \\
\text { months }\end{array}$ \\
\hline & & $71 / F$ & $\begin{array}{l}\text { Lumbosacral } \\
\text { radiculopathy }\end{array}$ & $\llcorner 4-5$ & $\begin{array}{l}\text { Biopsy of } \\
\text { right } L 5 \\
\text { nerve root }\end{array}$ & DLBCL & Primary & $\begin{array}{l}\text { Negative on } \\
\text { whole-body } \\
\text { PET/CT }\end{array}$ & $\begin{array}{l}\text { Positive } \\
\text { on CSF } \\
\text { cytology }\end{array}$ & $\begin{array}{l}\text { R-CHOP, } \\
\text { IT-MTX }\end{array}$ & - \\
\hline $\begin{array}{l}\text { Nakashima } \\
\text { et al. }\end{array}$ & 2014 & $59 / \mathrm{M}$ & $\begin{array}{l}\text { Lumbosacral } \\
\text { radiculopathy }\end{array}$ & T12-S1 & $\begin{array}{l}\text { Open biopsy } \\
\text { at L3-4 }\end{array}$ & DLBCL & Primary & $\begin{array}{l}\text { Negative on } \\
\text { whole-body } \\
\text { PET/CT }\end{array}$ & $\begin{array}{l}\text { Negative } \\
\text { on CSF } \\
\text { cytology }\end{array}$ & $\begin{array}{l}\text { HD-MTX, } \\
\text { RT } 50 \text { Gy }\end{array}$ & $\begin{array}{l}\text { Alive, } \\
\text { progression-free } \\
\text { for } 1 \text { year }\end{array}$ \\
\hline \multirow[t]{3}{*}{$\begin{array}{l}\text { Marquardt } \\
\text { and Li }\end{array}$} & 2018 & $73 / F$ & $\begin{array}{l}\text { Lumbosacral } \\
\text { radiculopathy }\end{array}$ & L2-S2 & $\begin{array}{l}\text { Open biopsy } \\
\text { at } L 2\end{array}$ & DLBCL & Primary & $\begin{array}{l}\text { Negative on } \\
\text { whole-body } \\
\text { PET/CT }\end{array}$ & $\begin{array}{l}\text { Negative } \\
\text { on CSF } \\
\text { cytology }\end{array}$ & $\begin{array}{l}\text { R-CHOP, } \\
\text { IT-MTX, } \\
\text { RT }\end{array}$ & $\begin{array}{l}\text { Alive, } \\
\text { progression-free } \\
\text { for } 20 \text { months }\end{array}$ \\
\hline & & $61 / M$ & $\begin{array}{l}\text { Sacral } \\
\text { radiculopathy }\end{array}$ & S1 & $\begin{array}{l}\text { Biopsy of } \\
\text { right S1 } \\
\text { nerve }\end{array}$ & $\mathrm{DLBCL}$ & Primary & $\begin{array}{l}\text { Negative on } \\
\text { whole-body } \\
\text { PET/CT }\end{array}$ & $\begin{array}{l}\text { Negative } \\
\text { on CSF } \\
\text { cytology }\end{array}$ & $\begin{array}{l}\text { R-CHOP, } \\
\text { IT-MTX, } \\
\text { RT }\end{array}$ & $\begin{array}{l}\text { Alive, } \\
\text { progression-free } \\
\text { for } 48 \text { months }\end{array}$ \\
\hline & & $47 / M$ & $\begin{array}{l}\text { Sacral } \\
\text { radiculopathy }\end{array}$ & S1-2 & $\begin{array}{l}\text { Biopsy of } \\
\text { right sacral } \\
\text { ala }\end{array}$ & DLBCL & Primary & - & $\begin{array}{l}\text { Negative } \\
\text { on CSF } \\
\text { cytology }\end{array}$ & $\begin{array}{l}\text { R-CHOP, } \\
\text { IT-MTX, } \\
\text { RT }\end{array}$ & $\begin{array}{l}\text { Alive, } \\
\text { progression-free } \\
\text { for } 13 \text { years }\end{array}$ \\
\hline $\begin{array}{l}\text { Sasaki } \\
\text { et al. }\end{array}$ & 2018 & $62 / M$ & $\begin{array}{l}\text { Lumbosacral } \\
\text { radiculopathy }\end{array}$ & L2-4 & $\begin{array}{l}\text { Open biopsy } \\
\text { at L3 }\end{array}$ & DLBCL & Primary & $\begin{array}{l}\text { Negative on } \\
\text { whole-body } \\
\text { PET/CT }\end{array}$ & $\begin{array}{l}\text { Negative } \\
\text { on CSF } \\
\text { cytology }\end{array}$ & $\begin{array}{l}\text { HD-MTX } \\
+ \text { R, RT } \\
30 \text { Gy }\end{array}$ & $\begin{array}{l}\text { Alive, } \\
\text { progression-free } \\
\text { for } 8 \text { months }\end{array}$ \\
\hline $\begin{array}{l}\text { Suzuki } \\
\text { et al. }\end{array}$ & 2018 & $65 / M$ & CES & LI-S1 & $\begin{array}{l}\text { Open biopsy } \\
\text { at L4-5 }\end{array}$ & DLBCL & Primary & $\begin{array}{l}\text { Negative on } \\
\text { whole-body } \\
\text { PET/CT }\end{array}$ & $\begin{array}{l}\text { Positive } \\
\text { on CSF } \\
\text { cytology }\end{array}$ & $\begin{array}{l}\text { HD-MTX } \\
\text { Ara-C }\end{array}$ & $\begin{array}{l}\text { Alive, } \\
\text { progression-free } \\
\text { for } 6 \text { years }\end{array}$ \\
\hline Ban et al. & 2019 & 64/M & CES & L3 & $\begin{array}{l}\text { Surgical } \\
\text { excision at } \\
\text { L3 }\end{array}$ & DLBCL & $\begin{array}{l}\text { Secondary } \\
\text { from } N H L \text { in } \\
\text { left inguinal } \\
L N, 7 \\
\text { months ago }\end{array}$ & $\begin{array}{l}\text { Negative on } \\
\text { whole-body } \\
\text { PET/CT }\end{array}$ & - & R-CHOP & $\begin{array}{l}\text { Deceased in } 13 \\
\text { months }\end{array}$ \\
\hline $\begin{array}{l}\text { Bourque } \\
\text { et al. }\end{array}$ & 2019 & $77 / M$ & $\begin{array}{l}\text { Lumbosacral } \\
\text { radiculopathy }\end{array}$ & S1-3 & - & - & $\begin{array}{l}\text { Secondary } \\
\text { from NL in } \\
\text { left ulnar } \\
\text { nerve, } 1 \\
\text { year ago }\end{array}$ & - & - & RT & $\begin{array}{l}\text { Decreased in few } \\
\text { months }\end{array}$ \\
\hline $\begin{array}{l}\text { Current } \\
\text { case }\end{array}$ & 2020 & $67 / F$ & CES & L3-S3 & $\begin{array}{l}\text { Surgical } \\
\text { excision at } \\
\text { L4-5 }\end{array}$ & DLBCL & Primary & $\begin{array}{l}\text { Negative on } \\
\text { whole-body } \\
\text { PET/CT }\end{array}$ & $\begin{array}{l}\text { Positive } \\
\text { on brain } \\
\text { MRI }\end{array}$ & R-MPV & - \\
\hline
\end{tabular}

M: male; F: female; CES: cauda equina syndrome; CSF: cerebrospinal fluid; DLBCL: diffuse large B-cell lymphoma; NHL: non-Hodgkin's lymphoma; LN: lymph node; NL: neurolymphomatosis; CT: computed tomography; PET: positron emission tomography; CNS: central nerve system; HD-MTX: high-dose methotrexate; Ara-C: arabinosyl cytosine; RT: radiation therapy; R-CHOP: rituximab, cyclophosphamide, doxorubicin, vincristine, and prednisone; IT-MTX: intrathecal methotrexate; R: rituximab; R-MPV: rituximab, high-dose methotrexate, procarbazine, and vincristine.

*Results are evaluated for systemic disease other than the spine lesion. 
unclear, a few cases have been shown a positive response to systemic chemotherapy ${ }^{9,10,12,14)}$.

\section{CONCLUSION}

To the best of our knowledge, our case is unique in that primary CNS lymphoma occurred in the cauda equina as the first manifestation. This rare, but potentially treatable, entity of malignancy is frequently misdiagnosed. Thus, timely diagnosis, with biopsy and multiple imaging modalities, is important for adequate treatment.

\section{CONFLICTS OF INTEREST}

No potential conflict of interest relevant to this article was reported.

\section{REFERENCES}

1. Baehring JM, Damek D, Martin EC, Betensky RA, Hochberg FH: Neurolymphomatosis. Neuro Oncol 5:104-115, 2003

2. Ban Y, Jing Z, Zou J: Multiple secondary cauda equina nonHodgkin's lymphoma: A case report and literature review. BMC Cancer 19:594, 2019

3. Bourque PR, Sampaio ML, Warman-Chardon J, Samaan S, Torres C: Neurolymphomatosis of the lumbosacral plexus and its branches: case series and literature review. BMC Cancer 19:1149, 2019

4. Broen M, Draak T, Riedl RG, Weber WE: Diffuse large B-cell lymphoma of the cauda equina. BMJ Case Rep [epub ahead of print, 2014. doi:10.1136/bcr-2014-205950]

5. Byun JM, Kim KH, Kim M, Kim TM, Jeon YK, Park JH, et al.: Diagnosis of secondary peripheral neurolymphomatosis: a multi- center experience. Leuk Lymphoma 58:2624-2632, 2017

6. Engelhard HH, Villano JL, Porter KR, Stewart AK, Barua M, Barker FG, et al.: Clinical presentation, histology, and treatment in 430 patients with primary tumors of the spinal cord, spinal meninges, or cauda equina. J Neurosurg Spine 13:67-77, 2010

7. Grisariu S, Avni B, Batchelor TT, van den Bent MJ, Bokstein F, Schiff D, et al.: Neurolymphomatosis: An International Primary CNS Lymphoma Collaborative Group report. Blood 115:50055011, 2010

8. Jung KW, Park KH, Ha J, Lee SH, Won YJ, Yoo H: Incidence of primary spinal cord, spinal meninges, and cauda equina tumors in Korea, 2006-2010. Cancer Res Treat 47:166-172, 2015

9. Marquardt RJ, Li Y: Lumbosacral radiculoplexopathy as the initial presentation of lymphoma: A report of 4 cases. J Clin Neuromuscul Dis 19:196-202, 2018

10. Nakashima H, Imagama S, Ito Z, Ando K, Kobayashi K, Ukai J, et al.: Primary cauda equina lymphoma: case report and literature review. Nagoya J Med Sci 76:349-354, 2014

11. Nishijima H, Akagi T, Ueno T, Kon T, Haga R, Funamizu Y, et al.: Lumbosacral nerve root lesion with malignant lymphoma. Neurol India 66:580-581, 2018

12. Sasaki R, Ohta Y, Yamada Y, Tadokoro K, Takahashi Y, Sato $\mathrm{K}$, et al.: Neurolymphomatosis in the cauda equina diagnosed by an open biopsy. Intern Med 57:3463-3465, 2018

13. Shree R, Goyal MK, Modi M, Gaspar BL, Radotra BD, Ahuja $\mathrm{CK}$, et al.: The diagnostic dilemma of neurolymphomatosis. J Clin Neurol 12:274-281, 2016

14. Suzuki K, Yasuda T, Hiraiwa T, Kanamori M, Kimura T, Kawaguchi Y: Primary cauda equina lymphoma diagnosed by nerve biopsy: A case report and literature review. Oncol Lett 16:623631, 2018

15. Teo MK, Mathieson C, Carruthers R, Stewart W, Alakandy L: Cauda equina lymphoma-a rare presentation of primary central nervous system lymphoma: case report and literature review. Br J Neurosurg 26:868-871, 2012 